\title{
Early rehabilitation in sepsis: a prospective randomised controlled trial investigating functional and physiological outcomes The i-PERFORM Trial (Protocol Article)
}

Geetha Kayambu ${ }^{1 * \dagger}$, Robert J Boots ${ }^{1,2}$ and Jennifer D Paratz ${ }^{1}$

\begin{abstract}
Background: Patients with sepsis syndromes in comparison to general intensive care patients can have worse outcomes for physical function, quality of life and survival. Early intensive care rehabilitation can improve the outcome in general Intensive Care Unit (ICU) patients, however no investigations have specifically looked at patients with sepsis syndromes. The 'i-PERFORM Trial' will investigate if early targeted rehabilitation is both safe and effective in patients with sepsis syndromes admitted to ICU.

Methods/Design: A single-centred blinded randomized controlled trial will be conducted in Brisbane, Australia. Participants ( $n=252$ ) will include those $\geq 18$ years, mechanically ventilated for $\geq 48$ hours and diagnosed with a sepsis syndrome. Participants will be randomised to an intervention arm which will undergo an early targeted rehabilitation program according to the level of arousal, strength and cardiovascular stability and a control group which will receive normal care.

The primary outcome measures will be physical function tests on discharge from ICU (The Acute Care Index of Function and The Physical Function ICU Test). Health-related quality of life will be measured using the Short Form36 and the psychological component will be tested using The Hospital Anxiety and Depression Scale. Secondary measures will include inflammatory biomarkers; Interleukin-6, Interleukin-10 and Tumour Necrosis Factor- $\alpha$, peripheral blood mitochondrial DNA content and lactate, fat free muscle mass, tissue oxygenation and microcirculatory flow.
\end{abstract}

Discussion: The 'i-PERFORM Trial' will determine whether early rehabilitation for patients with sepsis is effective at improving patient outcomes with functional and physiological parameters reflecting long and short-term effects of early exercise and the safety in its application in critical illness.

Trial Registration: Australia and New Zealand Clinical Trials Register (ANZCTR): ACTRN12610000808044

\section{Background}

Critical Illness and trauma are the primary sources of intensive care unit admissions. An estimated $2-11 \%$ of these patients require prolonged stays in the ICU which accounts for $25-45 \%$ of total ICU days [1]. Survivors of intensive care, especially those with prolonged admissions, may exhibit severe psychological and physical problems

\footnotetext{
* Correspondence: g.kayambu@uq.edu.au

† Contributed equally

'Burns, Trauma \& Critical Care Research Centre, School of Medicine, The

University of Queensland, Brisbane QLD 4029, Australia

Full list of author information is available at the end of the article
}

[2] and have a lower health-related quality of life up to one year following discharge from the hospital [3]. Almost a quarter of these patients are either admitted with or develop sepsis i.e. a severe, specific inflammatory response to infection, during the course of their stay in the ICU with major immediate and long-term effects on morbidity and mortality [4]. Patients with sepsis can have a worse outcome both in terms of overall functioning and mortality rates [5]. There is evidence that specific decreases in muscle mass and muscle force occur in sepsis syndromes caused by a variety of mechanisms [6]. This has major 
effects on the health and productivity of survivors as well as ultimately impact on the availability of ICU and hospital beds, surgical waiting lists, health costs and society.

Hypothesising that light to moderate exercise can partially reverse this condition without causing harm to critically ill patients, several exercise trials conducted in general intensive care patients have indicated positive findings that early intervention with exercise is able to prevent critical illness weakness syndromes, loss of muscle mass, decrease duration of mechanical ventilation, length of hospital and ICU stay and improve overall quality of life [7]. Specifically however, it is important to investigate early rehabilitation in patients with sepsis syndromes as the inflammatory process in sepsis can aggravate and accelerate the rate of muscle wasting in addition to the immobility associated with the disease in its early stages. It is equally important to ensure that early exercise in sepsis does not result in oxidative stress or cause substantial tissue injury potentially worsening the inflammatory reaction. The physiological reasons for improvement or adverse effects require investigation (Figure 1).

\section{Sepsis Syndrome}

Sepsis is a systemic inflammatory response associated with an infectious insult. It is the leading cause of death in critically ill patients and is often associated multi organ failure [8]. The inflammatory cytokines associated with sepsis such as Interleukin-6 (IL-6), Interleukin-10 (IL-10) and Interleukin-1 $\beta$ (IL-1 $\beta$ ) are found to be correlated with the severity of the disease, the evolution of organ failure as measured by the SOFA score and mortality [9]. Physiological responses to inflammation as described in Table 1 in addition to the presence of a suspected or proven infection has been an internationally accepted definition for "Sepsis" $[8,10]$. Increasing sepsis severity correlates with increasing mortality, rising from $25-30 \%$ for severe sepsis to $40-70 \%$ for septic shock [11].

\section{Systemic Inflammation and Proteolysis}

The aggressive inflammatory process that occurs during sepsis also affects muscle force, muscle mass and ultimately physical function [12,13]. Persistent elevation of circulating levels of Interleukin-6 (IL-6) have been known to infiltrate myocytes with inflammatory factors such as prostaglandins leading to proteolysis, myocyte degeneration, and muscle atrophy [14]. Tumour Necrosis factor- $\alpha$ (TNF- $\alpha)$ overexpression in sepsis is linked to the development of cachexia [15] through endothelial dysfunction, leading to myocyte apoptosis, reduction in skeletal muscle mass, weakness and myopathy. It has been hypothesised that the prevention of excessive release of pro-inflammatory cytokines [15], and activation of proteolytic pathways leading to limitation of free-radical generation [16] may inhibit the catabolic skeletal muscle changes in sepsis [17] and thus critical illness weakness syndromes.

\section{Inflammatory Biomarkers and Exercise}

Interleukin-10 (IL-10); an anti-inflammatory cytokine is thought to inhibit proteolysis [18] while low levels have been postulated to lead to excessive inflammation and muscle damage [19]. IL-10 is known to attenuate the

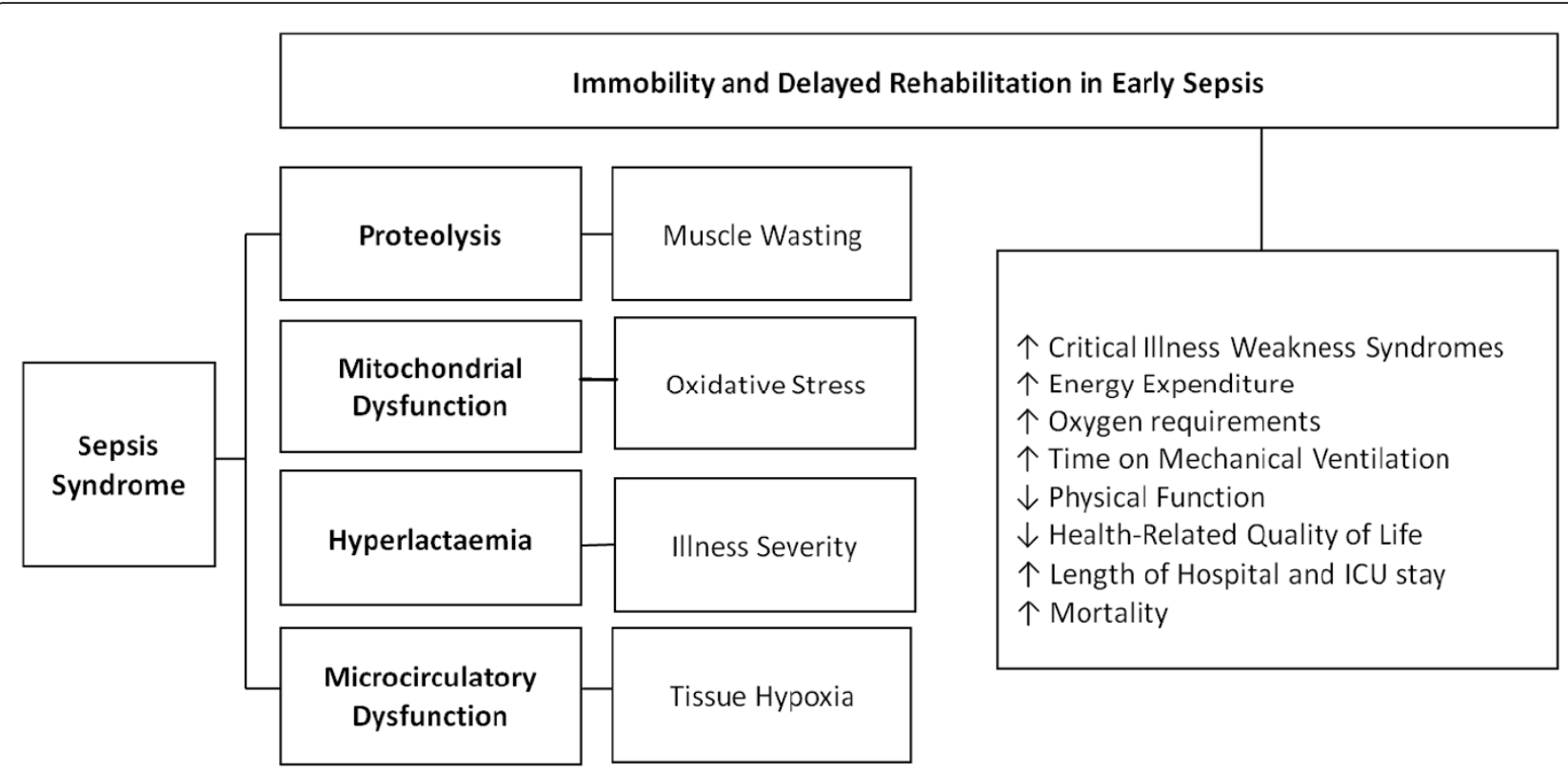

Figure 1 Impact of delayed ICU rehabilitation in early sepsis. This diagram illustrates the sequential impact of delayed rehabilitation for patients with sepsis as a result of the inflammatory processes and the detrimental short and long-term outcomes. 
Table 1 Criteria for SIRS and Sepsis syndromes (modified from Dellinger et al [57])

\begin{tabular}{|c|c|c|}
\hline Syndrome & Criteria & Mortality \\
\hline $\begin{array}{l}\text { Systemic inflammatory response syndrome (SIRS) any } \\
\text { two or more of the following criteria }\end{array}$ & 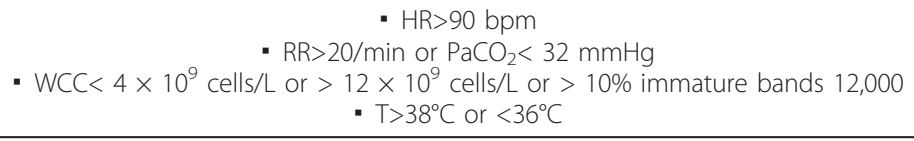 & \\
\hline Sepsis & - SIRS + proven infection & $15 \%$ \\
\hline Severe sepsis & - Sepsis + failure of one or more organs & $20 \%$ \\
\hline Septic shock & $\begin{array}{l}\text { - Severe sepsis + cardiovascular failure despite adequate fluid resuscitation } \\
\text { (usually SABP }<90 \mathrm{mmHg} \text { or }>40 \mathrm{mmHg} \text { from baseline) }\end{array}$ & $45 \%$ \\
\hline
\end{tabular}

HR; Heart rate, RR; Respiratory Rate; WCC; White Cell Count; T; Temperature, SIRS; Systemic Inflammatory Response Syndrome, SABP; Systolic Arterial Blood Pressure.

synthesis of TNF- $\alpha$ surface receptor and its suppressive effects may be beneficial in pathology that results from inflammatory dysregulation such as in sepsis.

A number of studies [20-22] in healthy and other diseased populations; such as in chronic heart failure, have shown that aerobic and resisted exercise alters proinflammatory cytokines specifically decreasing IL-6 and TNF- $\alpha$ and increasing IL-10. Simple physical exercises such as repetitive passive muscle stretches have been shown to decrease pro-inflammatory cytokine (IL-6), increase anti-inflammatory cytokine (IL-10) and improve the IL-10/TNF- $\alpha$ ratio in chronic critically ill patients [23]. The use of electrical muscle stimulation on major muscle groups has been shown to attenuate the production of TNF- $\alpha$ [24]. It is therefore important to investigate the effect of exercise on IL-10 in sepsis.

Our pilot study tested 20 patients with sepsis syndromes randomised into a treatment (proactive rehabilitation) and a control group. The trial demonstrated significant decreases $(-7.2 \%)$ in the percentage fat free mass in the control group with no decreases in the intervention group. Significant increases in IL-10 (mean difference 12.1 [SE $+/-2.1], \mathrm{p}<0.01)$ were found in the treatment group. Clinically relevant findings from this study indicate early exercise reduces loss of muscle mass in sepsis [25].

\section{Oxidative stress}

Oxygen-derived free radicals play an important role in the development and progression of disease in critically ill patients resulting in increases in the level of Reactive Oxygen Species (ROS) [26] or decreases in antioxidant defences [27] causing oxidative stress [28]. ROS can play a pivotal role in stimulating the inflammatory system by causing an increase in cytokines (e.g. Interleukins and TNF- $\alpha$ ). Both cytokines and ROS can enter the circulation and mediate systemic inflammatory responses linked with clinical conditions [29] which are interrelated in causing muscle proteolysis resulting in the induction of sepsis-induced myopathy [6].

In patients with sepsis, antioxidant depletion has been found to be associated with mitochondrial dysfunction where oxidative stress generates bioenergetic failure [30] which may affect changes in mitochondrial DNA (mtDNA) quantity [31], as well as increase mutations or deletions. This has been hypothesised to be part of the mechanism underlying multiple organ failure and death [32,33].

Oxidative stress also acts as an atrophic stimulus in an unloaded muscle [34] promoting wasting by modifying redox-sensitive processes in its muscle fibres such as during periods of disuse in locomotor skeletal muscles [35] and the unloaded diaphragm during prolonged mechanical ventilation $[36,37]$. Currently, however, there is no published data on the effects of exercise on oxidative stress on the critically ill population particularly in sepsis.

\section{IIIness Severity}

Hyperlactaemia is a marker of illness severity in sepsis $[38,39]$. During early sepsis, perturbation of glycolytic mechanisms can occur. In septic shock, high concentrations of circulating adrenaline can derive large quantities of lactate out of skeletal muscles, overwhelming lactate clearance mechanisms with resultant, hyperlactaemia $[40,41]$. The effect of light exercise on lactate clearance mechanisms in early sepsis remains to be investigated.

\section{Microcirculation and Muscle Oxygenation}

Sepsis impairs microcirculatory function causing tissue hypoxia [42]. This, combined with blood flow diversion from less important tissues to vital organs [43] promotes decreased muscular oxygen extraction [44]. However, the use of electrical stimulation [45] on major muscle groups and a physical exercise rehabilitation program in chronic heart failure patients [46] have demonstrated short-term beneficial systemic effects on microcirculation. The effect of early activity on microcirculation in patients with sepsis should be further investigated.

\section{Early Rehabilitation may Modulate Detrimental Effects of Sepsis}

The roles of pro-inflammatory cytokines, mitochondrial changes and muscle proteolysis in causing sepsisinduced myopathy in critically ill patients are 
hypothesised from animal models [6,14]. It is postulated that exercise can modulate cytokine levels [47], ROS production [48] and ATP levels [49] in patients with sepsis. In conjunction with an early mobility protocol, ambulation during mechanical ventilation in the early stages of critical illness is becoming widely practiced [50-52], especially with interruption of sedation [53-55]. Conservatively, simple passive movements [56] and neuromuscular electrical stimulation [45] as a precursor to active mobilisation can induce microcirculatory changes that may attenuate the anti-inflammatory effects in early sepsis.

Overall, early rehabilitation in critical illness seems to show reduced time on the ventilator, improved quality of life and reduced hospital and ICU stay [7] but this is not specific to sepsis. Furthermore, the short-term systemic effects of exercise for ICU patients remain highly speculative and debatable. The impact of exercise in the early stages of critical illness on effects such as oxidative stress and microcirculatory alterations have been unexplored and speculated from other populations. Exploring these physiological factors in response to exercise can contribute to determining the safety of early rehabilitation in sepsis.

\section{Hypotheses and Aims}

The primary research hypothesis of the "i-PERFORM Trial" is that patients with defined sepsis syndromes [57] in the ICU who participate in an early targeted rehabilitation program will have improved physical function and an improved quality of life. The secondary hypotheses is that there will be systemic effects underlying primary improvements which will include; increased fat free mass; decreased pro-inflammatory and increased anti-inflammatory cytokines; increased peripheral blood mitochondrial DNA content and reduced blood lactate levels; and improved tissue oxygenation and microcirculation flow.

\section{Methods/Design}

\section{Methods and Design}

The i-PERFORM Trial is a prospective double blinded randomised controlled trial (RCT) in patients with sepsis syndromes randomised into an early rehabilitation intervention arm or a control arm during the course of ICU stay. All outcomes will be measured by a blinded assessor from the research team. This study is being conducted in a quaternary level general Intensive Care Unit at the Royal Brisbane and Women's Hospital (RBWH), Brisbane, Queensland, Australia. The Human Research Ethics Committee at RBWH and the Medical Research Ethics Committee at The University of Queensland have approved this study. The study protocol is registered with the Australian New Zealand Clinical Trials Registry (ANZCTR). Figure 2 illustrates the methodology design for this RCT through recommendations from CONSORT (Consolidated Standards of Reporting Trials) Statement.

\section{Inclusion Criteria}

To be eligible for recruitment in the study, participants must be aged 18 years and above; ventilated for $\geq 48$ hours; diagnosed with a sepsis syndrome or have a high clinical suspicion of sepsis [57]. Patients with head injuries, burns, spinal injuries, and multiple fractured lower limbs requiring specific rehabilitation regimes and patients with septic shock who are unresponsive to maximal treatment and those who are moribund or have an expected mortality within 48 hours will be excluded.

\section{Recruitment, Randomisation and Blinding}

Eligible patients will be identified and consent will be sought by the chief and principal investigators, with next of kin or substitute decision maker. Participants recruited will be randomized into control and intervention arms using computer generated randomization; http://www. randomization.com/. The randomization sequence will be generated and re-identifiable serial numbers will be assigned by a research personnel not involved in the study. They will be concealed from consent designee research staff for group allocation to prevent selection bias and protected by an electronic password. Participants, substitute decision makers and outcome assessors will be blinded to group allocation to avoid influence over treatment effect. Blinding of the treating physiotherapist and health care providers cannot be ensured due to the nature of the research intervention.

\section{Demographics}

Patient demographic information will be collected at baseline (recruitment) and ICU and hospital discharge and will include; age, gender, primary reason for readmission to ICU, Acute Physiological and Chronic Health Evaluation II (APACHE II)[58], Sequential Organ Failure Assessment (SOFA) score [59], Charlson Comorbidity Index [60], time on mechanical ventilation, length of ICU and hospital stay, readmissions to ICU and 90-day mortality.

\section{Intervention Arm}

Participants randomised to the intervention arm of the trial will undergo a specific targeted rehabilitation program prescribed by the treating ICU physiotherapist for 30 minutes, one to two times daily until discharge from the ICU within 48 hours of the diagnosis of sepsis (Early Intervention Arm). The rehabilitation program will comprise of passive or active range of motion exercises, resistive exercises, electrical muscle stimulation to major muscle groups, leg or arm ergometry, sitting out of bed, tilt table therapy and ambulation with assistance. 


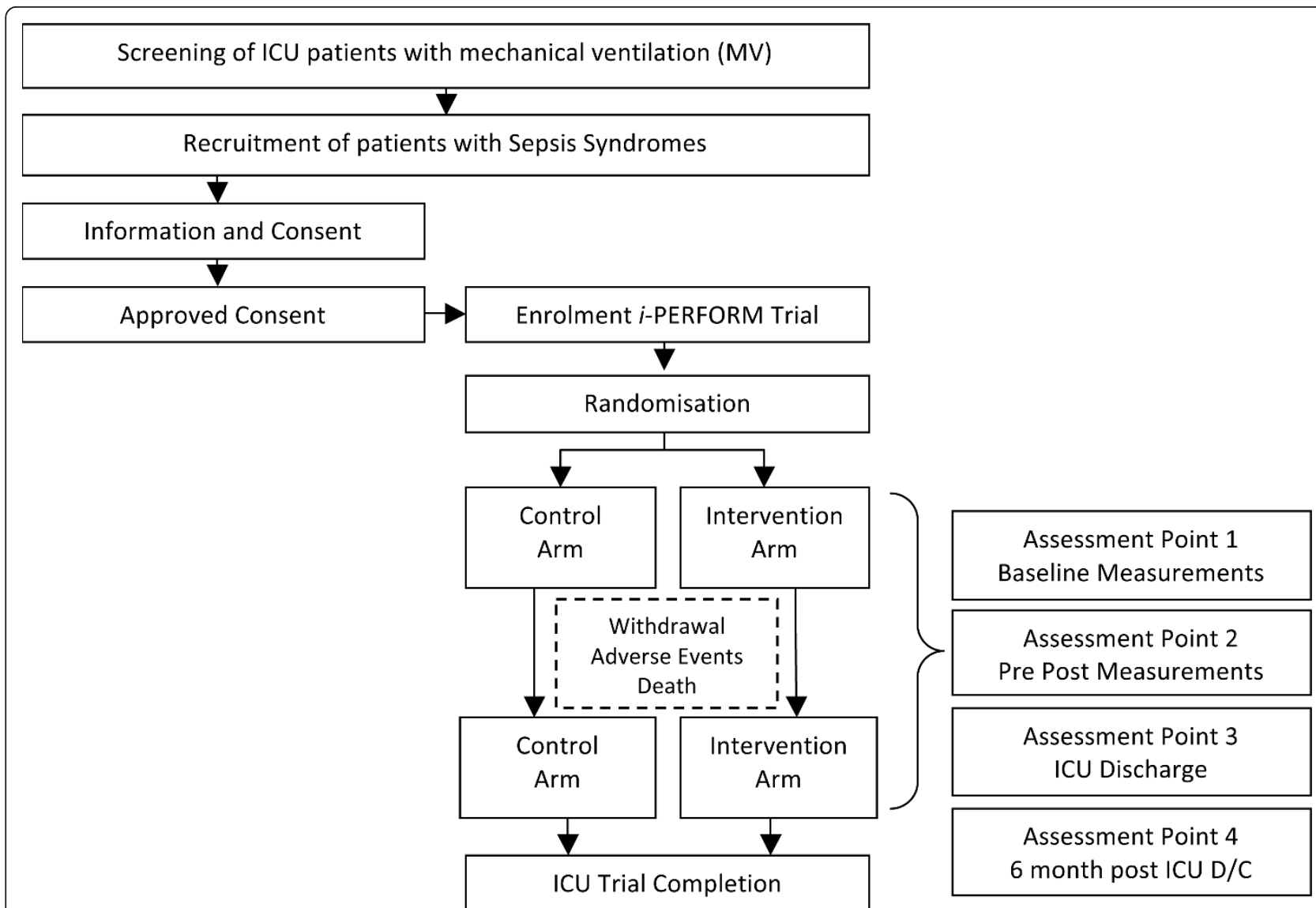

Figure 2 Research methodology sequence diagram. The inclusion criteria are as follows: Age $\geq 18$ yrs and remain ventilated for $\geq 48$ hours; present with documented sepsis or high clinical suspicion of sepsis. Patients with head injuries, burns, spinal injuries, and multiple fractured lower limbs, those with septic shock unresponsive to treatment, moribund or with expected mortality within 48 hours will be excluded.

The rehabilitation strategy for the participants in the intervention arm has been carefully planned with consideration for different levels of cardiovascular stability, ability of the patient to co-operate, and existing evidence regarding rehabilitation techniques and mobilisation strategies in intensive care. It will be administered and progressed by the discretion of the physiotherapist, according to individual acuity of illness and level of cooperation as based on the Ramsay sedation score which will account for the intervention progression stages. If the patient deteriorates, a lower level of activity will be given. Table 2 summarises the intervention strategies.

\section{Control Arm}

Participants allocated to the control arm will receive standard ICU care. The participants randomised to this group will not receive active rehabilitation from the research team, i.e. will not be given targeted exercises early in their disease process upon recruitment. However, they will continue to receive simple and less regular mobilisation activities from other sources, as part of the usual ICU care such as sitting out of bed or ambulation immediately prior to discharge from the unit. The time involved in these activities and the type of activities performed will be recorded and compared between groups.

\section{Safety and Withdrawal criteria}

A safety audit will be completed on patients in the rehabilitation group to monitor any adverse events [61] during rehabilitation. Data will be gathered from the patient's IntelliVue bedside monitor MP70 (Phillips) every 10 seconds and printed out for 10 minutes prior, during and post rehabilitation. The intra-arterial line will be zeroed 10 minutes prior to exercise. A withdrawal criterion with a checklist of adverse events will be used for the clinical decision of withdrawal or modification of exercise intervention.

\section{Primary Outcome Measures}

All primary outcomes will be measured at baseline (recruitment) and at ICU discharge and quality of life at 6 months post hospital discharge. The primary outcome 
Table 2 Intervention Strategies for early targeted rehabilitation for the critically ill.

\begin{tabular}{|c|c|c|c|c|}
\hline $\begin{array}{l}\text { Ramsay Sedation } \\
\text { Scale }\end{array}$ & $\begin{array}{l}\text { Stages of } \\
\text { exercise } \\
\text { progression }\end{array}$ & Conditions & Type of Intervention & $\begin{array}{l}\text { Recommended Exercise } \\
\text { Prescription }\end{array}$ \\
\hline \multirow[t]{2}{*}{$6-4$} & Stage 1 & Sedated & $\begin{array}{l}\text { Passive Range of Motion with stretch reflex to upper } \\
\text { and lower limbs }\end{array}$ & 30 Mins/day \\
\hline & & & Electrical Muscle Stimulation to major muscle groups & 30 Mins/day \\
\hline \multirow[t]{4}{*}{$3-2$} & Stage 2 & $\begin{array}{l}\text { Inotropic } \\
\text { Dependence }\end{array}$ & $\begin{array}{l}\text { Active Range of Motion/Lightly resisted with upper and } \\
\text { lower limb }\end{array}$ & 10 Mins $\times 2 /$ day \\
\hline & & & Electrical Muscle Stimulation to major muscle groups & 30 Mins/day \\
\hline & & & Sitting up in Bed with assistance as tolerated & 30 Mins $\times 2 /$ day \\
\hline & & & Sitting on Edge of Bed & 10 Mins $\times 2 /$ day \\
\hline \multirow[t]{6}{*}{2} & Stage 3 & Weight & $\begin{array}{l}\text { Active Range of Motion/Lightly resisted with upper and } \\
\text { lower limb }\end{array}$ & 10 Mins $\times 2 /$ day \\
\hline & & Bearing & Electrical Muscle Stimulation to major muscle groups & 30 Mins/day \\
\hline & & Muscle Strength & Sitting On Edge of Bed & 10 Mins $\times 2 /$ day \\
\hline & & Grade $<3$ & Sitting Out of Bed with assistance & 60 Mins $\times 2 /$ day \\
\hline & & & Lower Limb Ergometry & 15 Mins $\times 2 /$ day \\
\hline & & & Tilt Table Therapy & 30 Mins/day \\
\hline \multirow[t]{7}{*}{2} & Stage 4 & Weight & $\begin{array}{l}\text { Active Range of Motion/Lightly resisted with upper and } \\
\text { lower limb }\end{array}$ & 10 Mins $\times 2 /$ day \\
\hline & & Bearing & Electrical muscle stimulation to major muscle groups & 30 Mins/day \\
\hline & & Muscle Strength & Sitting On Edge of Bed & 10 Mins/day \\
\hline & & Grade $>3$ & Sitting Out of Bed & 30 Mins/day \\
\hline & & & Upper Limb Ergometry (low level resistance) & 15 Mins/day \\
\hline & & & Lower Limb Ergometry & 15 Mins $\times 2 /$ day \\
\hline & & & Ambulation with assistive device and therapist & 30 Mins $\times 2 /$ day \\
\hline
\end{tabular}

The Intervention strategies act as a guide for the execution of early exercises for the patients with sepsis syndromes based on the level of alertness according to the Ramsay Sedation Scale

measures will report the level of physical functioning and quality of life (long-term effects) of the participants. Physical function will be measured using the Acute Care Index of Function (ACIF) and the Physical Function ICU Test (PFIT).

The ACIF will essentially rate simple physical function (transfer bed to chair, sit to stand independently) of the patient on leaving the ICU [62]. The PFIT is a newly developed clinical tool for testing functional strength and endurance capacity in ICU patients. It consists of a battery of tests assessing endurance, muscle strength, cardiovascular capacity and functional ability which are domain representative of physical function and specific for the use of higher functional assessment in the critically ill [63]. These have been chosen as corresponding measuring instruments as they have demonstrated reliability, validity and responsiveness in the ICU population $[63,64]$.

Quality of life will be measured using the Short Form36 (SF-36) Health Survey Questionnaire. Information from the next of kin will be used to provide proxy scores which have been demonstrated to have good reliability and validity [65].

The constructs of psychological well being will be measured using the anxiety subscale of the Hospital
Anxiety and Depression Scale (HADS) as psychological wellbeing is known to be highly correlated to functional physical outcomes [66].

\section{Secondary Outcome Measures}

The secondary outcome measures will report on the physiological factors (short-term effects) illustrating underlying mechanisms of early intervention undertaken in the study. Blood samples $(8 \mathrm{ml})$ will be obtained from the patients' arterial line, for the analysis of cytokines, blood lactate and mtDNA levels pre and post intervention during the trial. All blood samples taken will be centrifuged (Spintron GT-25E/LL, Australia) for 15 minutes at 3000 rpm, within 20 minutes of collection. Plasma from the whole blood for the cytokine analysis and peripheral blood mononuclear cells (PBMC) forming the buffy coat pellet for DNA isolation will be stored at $-80^{\circ} \mathrm{C}$.

\section{Inflammatory Biomarkers}

Biomarkers will be measured before intervention and 30 minutes post intervention daily (week 1) and thereafter twice weekly (till ICU discharge). The acute inflammatory response of early exercise in sepsis will be measured by changes in levels of pro-inflammatory cytokines (IL- 6 and TNF- $\alpha$ ) and anti-inflammatory cytokine (IL-10). IL-6, IL-10 and TNF- $\alpha$ will be measured 
from plasma samples with the Milliplex cytokine panels from Millipore (Billerica, MA, USA) using a Luminex 100 assay, with inter and intra-assay $\mathrm{CV}<7 \%$.

\section{Muscle Mass}

Fat free mass will account for muscle mass (lean tissue) measured at baseline (week 0) and weekly thereafter (till ICU discharge) using the Multi-Frequency Bioelectrical Impedance Spectroscopy (BIS) Machine (ImpediMed SFB7, ImpediMed Ltd, Brisbane, Australia). Measurements will be taken at a standardised time of the day as practicable for validity [67] and at standardised anatomical landmarks for reproducibility of results [68]. Pairs of gel electrodes will be placed on the hand and foot on the right side of the body with the participant positioned in supine. ICU monitoring will continue during measurement without interference [69]. An estimate will be made of the critically ill patients' fluid balance. Urinary catheters will be emptied, prior to measurement. If subjects are known to have ascites, a pleural effusion or a renal replacement therapy, measurements will not be done. Percentage fat free mass will be measured in triplicate and analysed to reflect if proteolysis had occurred and resulted in the loss of muscle mass [70].

\section{Oxidative Stress Markers}

Oxidative stress will be determined through changes in mitochondrial DNA levels. Mitochondrial DNA copy number in the PBMC will be measured by determining relative amounts of mitochondrial to nuclear DNA using quantitative real-time PCR. Mitochondrial DNA (mtDNA) levels in the peripheral blood will be measured at baseline (week 0) and weekly thereafter (till ICU discharge). Total DNA from the blood sample will be extracted from the buffycoat using a DNA Analysis Kit (Machery Nagel Blood XL, Germany) and mtDNA quantity will be analysed using SYBR Green Master Mix Real Time PCR kit (Applied Biosystems, Warrington, United Kingdom). Triplicate analysis of blood samples will be conducted and trends of longitudinal increases in mitochondrial DNA levels will be reported.

\section{IIIness Severity}

Blood lactate levels will be measured before intervention and 30 minutes post intervention with arterial blood using a standard benchtop Arterial Blood Gas Analyser (ABL 700 Series gas Machines, RADIOMETER, Copenhagen, Denmark). Lactate concentration trends will be used to analyse lactate clearance rates [71] as a marker of illness severity.

\section{Muscle oxygenation and Microcirculation}

Muscle oxygenation and microcirculatory changes will be measured using the Near Infrared Oxygenation (NIR0) Monitor and the Orthogonal Polarization Spectral (OPS) Microscan respectively.

Muscle (tissue) oxygenation will be measured pre and post intervention daily using the NIRO (NIRO-200,
Hamamatsu, Japan) with a probe placed on a peripheral muscle to detect levels of muscle oxygenation $\left(\mathrm{StO}_{2}\right)$ following acute exercise [72]. Muscle oxygen consumption will be further analysed through induced ischemia and active hyperaemia [73]. A pneumatic cuff will be placed above the elbow and inflated to $50 \mathrm{mmHg}$ above the patient's systolic blood pressure and the occlusion will be retained for 3 minutes inducing local ischemia. $\mathrm{StO}_{2}$ will be recorded continuously for 3 minutes before, during and after arterial occlusion [44].

Improvements in microcirculation following acute exercise will be measured pre and post intervention session once weekly using the OPS MicroScan (Microvision Medical Inc, Wallingford, PA, USA) with a non-invasive probe placed in the sub-lingual space to detect functional capillary density and blood flow velocity as indicators of microcirculatory function [74] using video images. Five video sequences per patient will be graded by 3 independent observers and assigned a flow velocity score to each of the 4 quadrants of each image [75]. Capillary density will be calculated using superimposed calibrated grid of vertical and horizontal lines on the images of number of small $(<20 \mu \mathrm{m})$ vessels crossing the lines of the grid divided by the total length of the lines, yielding the number of small vessels per millimetre [76]. An average of these 5 readings will be calculated for discrete capillary density value for each time point at which imaging was performed.

\section{Confounding Variables}

Critical illness neuropathy and myopathy is a possible feature of critically ill patients. Formal tests to establish critical illness neuropathy or myopathy can be painful, invasive, time consuming and expensive [6]. Such formal diagnosis may not implicate any pertinent findings in this trial. Basic physiotherapy assessment muscle testing done as part of regular intervention assessment using the Medical Research Council or Manual Muscle Test will detect any possible development of weakness without requirements for formal diagnosis and is accepted as configuration of critical illness polyneuromyopathy [77].

\section{Sample size}

Clinically important difference and the standard deviation estimates used in our sample size calculations were based on a previous clinical trial [64]. Sample size was calculated using the ACIF [62] for hypothesis testing with a type I error rate of 0.05 and 0.025 with Bonferroni adjustment and type II error rate of 0.20 (80\% power). A minimum of 35 per group (70 total) is required to detect a minimum clinical difference (effect size) of 0.7 for physical functional outcomes which will yield clinically significant results for the main hypothesis 
achievable by intervention. Projection of the sample size after adjusting for attrition or withdrawal rates, participation refusals and possible death due to sepsis; based on admission and mortality rates in the RBWH ICU, 126 patients per group (252 in total) will be required.

A smaller number of patients will undergo mtDNA, tissue oxygenation and microcirculatory measurements as these will be tested as pilot investigations. To test these secondary hypotheses, a minimum of 58 per group is required to detect a minimum clinical difference of 0.8 for clinically significant changes in mitochondrial DNA [78], a minimum 91 per group is required to detect a clinical difference of 0.4 and 59 per group is required to detect a minimum clinical difference of 0.7 for clinically significant changes in tissue oxygenation [45] and microcirculation [46] respectively, all corrected for with Bonferroni adjustment of 0.025 .

\section{Data Management and Statistical Analysis}

A baseline comparison using both student t-tests and chi-square for equal proportions of demographic data will be done between groups at enrolment. Data will be analyzed using SPSS Version 17.0. The distribution, range of scores and heterogeneity will be examined for participants in both groups. Mean changes scores, standard deviations and 95\% confidence intervals will be calculated and specific analysis detailed under each outcome measure will be performed. Repeated measures, between/within analysis of variance (ANOVA) will be performed to investigate for the main effects of time and group. Post hoc analyses will then be performed to ascertain where the differences occur. A Bonferroni correction will be used as the post hoc measure as there are multiple outcome measures. Analysis will be by both intention-to-treat and per-protocol method. Any violations of the protocol will be noted. Statistical significance will be set at $\mathrm{p}<0.05$ and $\mathrm{p}<0.025$ (two-tailed). All efforts will be taken to avoid missing data but if it occurs a carry forward imputation will be done and linear mixed modelling will be used for overall analysis. Reasons for loss of follow up will be recorded.

\section{Discussion}

The i-PERFORM Trial is an original study in investigating early rehabilitation in patients presenting with sepsis syndromes. Fully powered to be a larger trial from the pilot study, this RCT has been modified to include detailed and intensive interventional measures and further outcome measures; SF-36, PFIT, blood lactate, mitochondrial DNA, muscle oxygenation and microcirculation dynamics.

Controversies still exist with regards to the implementation of early exercise in critically ill patients despite growing evidence in the literature $[7,79]$. Introducing early rehabilitation in patients with sepsis syndromes is challenging and the direct physiological implications are unknown. Novel findings from the i-PERFORM Trial involving physiological markers of oxidative stress and microcirculation will translate better understanding of the short-term systemic effects of early exercise in critical illness. The mechanisms and associations between early exercise and the inflammatory effects of sepsis explored in this trial will implicate on future rehabilitation management of patients with sepsis in intensive care units.

\section{Key Messages}

- The i-PERFORM RCT will determine whether early targeted rehabilitation will achieve a higher functional level and an improved quality of life in patients with sepsis.

- Participants in the intervention arm will receive early, targeted, individualised rehabilitation program comprising of passive, active and resisted exercises, electrical stimulation to major lower limb muscles, sitting out of bed with exercises and tilt-tabling to improve orthostatic reflexes, ergometry exercises for upper and lower limbs and ambulation.

- Physical functional capacity, quality of life, psychological wellbeing, inflammatory biomarkers, oxidative stress markers, illness severity, muscle oxygenation and microcirculation will be assessed using standard instruments at specific times before, during and after exercise sessions.

- Short-term systemic findings from early rehabilitation in sepsis will be novel and will translate better understanding of the acute effects of early exercise in critical illness.

\section{Abbreviations}

ACIF: Acute Care Index of Function; ANOVA: Analysis of Variance; ANZCTR Australian New Zealand Clinical Trials Registry; APACHE II: Acute Physiological and Chronic Health Evaluation II; ATP: Adenosine TriPhospate; BIS: Bioelectrical Impedance Spectroscopy; CONSORT: Consolidated Standards of Reporting Trials; CV: Coefficient of Variation; HADS: Hospital Anxiety and Depression Scale; ICU: Intensive Care Unit; IL-6: Interleukin-6; IL10: Interleukin-10; mtDNA: Mitochondrial DNA; NIRO: Near Infrared Oxygenation; OPS: Orthogonal Polarisation Spectral Microscan; PCR: Polymerase Chain Reaction; PFIT: Physical Function ICU Test; PBMC Peripheral Blood Mononuclear Cells; RBWH: Royal Brisbane and Women's Hospital; RCT: Randomised Controlled Trial; ROS: Reactive Oxygen Species; SF-36: Short Form-36 Health Questionnaire; SOFA: Sequential Organ Failure Assessment; $\mathbf{S t O}_{2}$ : Tissue/Muscle Oxygenation; TNF-a: Tumour Necrosis Factor alpha.

\section{Acknowledgements}

GK is supported by a Singaporean Postgraduate Award. The project is supported in kind by the Burns, Trauma and Critical Care Research Centre. We would like to thank the Royal Brisbane and Women's Hospital and The University of Queensland Centre for Clinical Research for the support of facilities to conduct this research. 


\section{Author details}

'Burns, Trauma \& Critical Care Research Centre, School of Medicine, The University of Queensland, Brisbane QLD 4029, Australia. ²Department of Intensive Care Medicine, The Royal Brisbane and Women's Hospital, Brisbane QLD 4029 Australia.

\section{Authors' contributions}

All authors contributed to the study design and methods, and the development of the grant application. JDP specifically contributed to initial conception of the project, pilot data and exercise strategies. GK and RJB contributed to the extended concept of the project for secondary measures. JDP and GK contributed to the statistical methods and power calculations. GK drafted the manuscript and all other authors critically revised it for important intellectual content. All authors approved the final version of the manuscript for publication.

\section{Authors' information}

Ms Geetha Kayambu, BSc Phyt (Hons), Physiotherapist, PhD Candidate The University of Queensland, School of Medicine, Burns Trauma \& Critical Care Research Centre, Brisbane OLD 4029, Australia

Contact Address:

The Royal Brisbane and Women's Hospital, Level 7, Block 6, Herston, Brisbane QLD 4029, Australia

Associate Professor Robert J Boots, MBBS (Hons), PhD, MMedSci Clin Epi $_{\text {. }}$ MHAIS, FRACP, FJFICM

Deputy Director

The Royal Brisbane and Women's Hospital, Department of Intensive Care Medicine, Burns Trauma \& Critical Care Research Centre, Brisbane QLD 4029 Australia

Dr. Jennifer D Paratz, FACP, Mphty, PhD, Grad Cert Ed (Medical and Health Sciences)

Research Fellow and Chair

Burns Trauma \& Critical Care Research Centre, The University of Queensland, School of Medicine, Brisbane OLD 4029, Australia

\section{Competing interests}

The authors declare that they have no competing interests.

Received: 18 July 2011 Accepted: 31 October 2011

Published: 31 October 2011

\section{References}

1. Williams TA, Ho KM, Dobb GJ, Finn JC, Knuiman M, Webb SAR: Effect of length of stay in intensive care unit on hospital and long-term mortality of critically ill adult patients. Br J Anaesth 2010, 104(4):459-464

2. Hurel D, Loirat P, Saulnier F, Nicolas F, Brivet F: Quality of life 6 months after intensive care: results of a prospective multicentre study using a generic health status scale and a satisfaction scale. Intensive Care Med 1997, 23:331-337.

3. Pettila $V$, Kaarlola A, Mäkeläinen $A$ : A health related quality of life of multiple organ dysfunction patients after one year after intensive care. Intensive Care Med 2000, 26:1473-1479.

4. Brun-Buisson C, Doyon F, Carlet J, Dellamonica P, Gouin F, Lepoutre A, Mercier J, Offenstadt G, Régnier B: Incidence, risk factors, and outcome of severe sepsis and septic shock in adults. A multicentre prospective study in intensive care in adults. JAMA 1995, 274(12):968-74

5. Heyland D, Hopman W, Coo H, Tranmer J, McColl M: Long term health related quality of life in survivors of sepsis. Short Form 36: a valid and reliable measure of health related quality of life. Crit Care Med 2001 28.3599-3605

6. Callahan LA, Supinski GA: Sepsis-induced myopathy. Crit Care Med 2009, 37(10):S354-367.

7. Kress JP: Clinical trials of early mobilization of critically ill patients. Crit Care Med 2009, 37(10 Suppl):S442-447.

8. Lever A, Mackenzie I: Sepsis: Definition, epidemiology and diagnosis. BMJ 2007, 335:879-932

9. Bozza F, Salluh J, Japiassu A, Soares M, Assis E, Gomes R, Bozza M, CastroFaria-Neto $\mathrm{H}$, Bozza P: Cytokine profiles as markers of disease severity in sepsis: a multiplex analysis. Crit Care 2007, 11(2):R49..

10. Bone R, Sibbald W, Sprung C: The ACCP-SCCM consensus conference on sepsis and organ failure. Chest Surg Clin N Am 1992, 101(6):1481-1483.
11. Lever A, Mackenzie I: Management of Sepsis. BMJ 2007, 335:929-932.

12. Winkelman C: Inactivity and Inflammation: Selected cytokines as biologic mediators in muscle dysfunction during critical illness. AACN Clin Issues 2004, 15(1):74-82.

13. Eikermann $M$, Koch $G$, Gerwig M: Muscle force and fatigue in patients with sepsis and multi-organ failure. Intensive Care Med 2006, 32:251-259.

14. Fink $H$, Helming M, Unterbuchner C, Lenz A, Neff F, Martyn JA, Blobner M: Systemic inflammatory response syndrome increases immobility-induced neuromuscular weakness. Crit Care Med 2008, 36(3):910-916.

15. Sharma R, Anker S: Cytokines, apoptosis and cachexia: The potential for TNF antagonism. Int J Cardiol 2002, 85:161-171.

16. Goodyear-Bruch and Pierce JD: Oxidative stress in critically ill patients. American Journal Critical Care 2002, 11:543-551.

17. Klaude M, Fredriksson K, Tjäder I, Hammarqvist F, Ahlman B, Rooyackers $O$ Wernerman J: Proteasome proteolytic activity in skeletal muscle is increased in patients with sepsis. Clin Sci 2007, 112:499-506.

18. Oberholzer A, Oberholzer C, Moldawer L: IL-10: A complex role in the pathogenesis of sepsis syndromes and its potential as an antiinflammatory drug. Crit Care Med 2002, 30:S58-S63.

19. Lyons A, Goelbel A, Mannick J, Lederer J: Protective effects of early interleukin-10 antagonism on injury-induced immune dysfuntion. Arch Surg 1999, 134:1317-1324.

20. Gielen S, Adams V, Möbius-Winkler S, Linke A, Erbs S, Yu J, Kempf W, Schubert A, Schuler G, Hambrecht R: Anti-inflammatory effects of exercise training in the skeletal muscles of patients with chronic heart failure. $J$ Am Coll Cardiol 2003, 42:861-868.

21. Vivodtzev I, Pépin J, Vottero G, Mayer V, Porsin B, Lévy P, Wuyam B Improvement in quadriceps strength and dyspnea in daily tasks after 1 month of electrical stimulation in severely deconditioned and malnourished COPD. Chest 2006, 129:1540-1548.

22. Nuhr M, Pette $D$, Berger $R$, Quittan M, Crevenna R, Huelsman $M$ Wiesinger G, Moser P, Fialka-Moser V, Pacher R: Beneficial effects of chronic low-frequency stimulation of thigh muscles in patients with advanced chronic heart failure. Eur Heart J 2004, 25:136-143.

23. Winkelman B: Cytokines in chronically ill patients after activity and rest. Bio Res Nurs 2007, 8:261-271.

24. Karavidas A, Raisakis K, Parissis J, Tsekoura D, Adamopoulos S, Korres D, Farmakis D, Zacharoulis A, Fotiadis I, Matsakas E, Zacharoulis A: Functional electrical stimulation improves endothelial function and reduces peripheral immune responses in patients with chronic heart failure. Eur $J$ Cardiovasc Prev Rehabil 2005, 13:592-597.

25. Paratz J, Lipman J, Boots R, Lingwood B: Early exercise attenuates inflammation and loss of muscle mass. Australian and New Zealand Intensive Care Society, Perth 2009.

26. Oldham K, Bowen P: Oxidative stress in critical care: Is antioxidant supplementation beneficial? J Am Diet Assoc 1998, 98:1001-1008.

27. Schorah C, Downing C, Piripitsi A, Gallivan L, Al-Hazaa A, Sanderson M, Bodenham A: Total Vitamin C, ascorbic acid and dehyroascorbic acid concentrations in plasma in critically ill patients. Am J Clin Nutr 1996, 63:760-765.

28. Powers S, Jackson M: Exercise-induced oxidative stress: cellular mechanisms and impact on muscle force production. Physiol Rev 2008, 88(4):1243-1276.

29. Espat N, Nelson W: Oxygen free radicles, oxidative stress and antioxidants in critical illness. Support Line 2000, 22:11-20.

30. Callahan L, Stofan D, Szweda L, Nethery D, Supinski G: Free radicals alter maximal diaphragmatic mitochondrial oxygen consumption in endotoxin-induced sepsis. Free Radic Biol Med 2001, 30:129-138.

31. Crouser $E$ : Mitochondrial dysfunction in septic shock and multiple organ dysfunction syndrome. Mitochondrion 2004, 4(5-6):729-741.

32. Brealey $D$, Singer $M$ : Mitochondrial dysfunction in sepsis. Curr Infect Dis Rep 2003, 5:365-371.

33. Brealey D, Brand M, Hargreaves I, Heales S, Land J, Smolenski R, Davies N, Cooper C, Singer M: Association between mitochondrial dysfunction and severity and outcome of septic shock. Lancet 2002, 360:219-223.

34. Powers S, Kavazis A, McClung J: Oxidative stress and disuse muscle atrophy. J Appl Physiology 2007, 102:2389-2397.

35. Kondo H, Nakagaki I, Sasaki S, Hori S, Itokawa Y: Mechanism of oxidative stress in skeletal muscle atrophied by immobilization. Am J Physiol Endocrinol Metab 1993, 265:E839-E844. 
36. Shanely R, Zergeroglu M, Lennon $S$, Sugiura T, Yimlamai T, Enns D, Belcastro A, Powers S: Mechanical ventilation-induced diaphragmatic atrophy is associated with oxidative injury and increased proteolytic activity. Am J Respir Crit Care Med 2002, 166:1369-1374.

37. Zergeroglu M, McKenzie M, Shanely R, Van Gammeren D, DeRuisseau K, Powers S: Mechanical ventilation-induced oxidative stress in the diaphragm. J Appl Physiol 2003, 95:1116-1124.

38. Revelly J, Tappy L, Martinez A, Bollmann M, Cayeux M, Berger M, Chioléro R: Lactate and glucose metabolism in severe sepsis and cardiogenic shock. Crit Care Med 2005, 33(10):2235-2240.

39. Valenza F, Aletti G, Fossali T, Chevallard G, Sacconi F, Irace M, Gattinoni L: Review. Lactate as a marker of energy failure in critically ill patients: Hypothesis. Crit Care 2005, 9(6):588-593.

40. Daniel A, Shizga IH, MacLean L: The anatomic and metabolic source of lactate in shock. Surg Gynecol Obstet 1978, 147(5):697-700.

41. Luchette F, Friend L, Brown C, Upputuri R, James J: Increased skeletal muscle Na+, K+-ATPase activity as a cause of increased lactate production after hemorrhagic shock. J Trauma 1998, 44(5):796-801.

42. Hammed S, Aird W, Cohn S: Oxygen delivery. Crit Care Med 2003, 31(12 Supp):S658-667.

43. Trzeciak S, Dellinger R, Parrillo J, Guglielmi M, Bajaj J, Abate N, Arnold R, Colilla S, Zanotti S, Hollenberg S, Microcirculatory Alterations in Resuscitation and Shock Investigators: Early microcirculatory perfusion derangements in patients with severe sepsis and septic shock: relationship to hemodynamics, oxygen transport, and survival. Ann Emerg Med 2007, 49:88-98.

44. Cretuer J: Muscle StO2 in critically ill patients. Current Opinion in Critical Care 2008, 14:361-356.

45. Gerovasili V, Tripodaki E, Karatzanos E, Pitsolis T, Markaki V, Zervakis D, Routsi C, Roussos C, Nanas S: Short-term systemic effect of electrical muscle stimulation in critically ill patients. Chest 2009, 136:1249-1256.

46. Gerovasili V, Drakos S, Kravari M, Malliaras K, Karatzanos E, Dimopoulos S, Tasoulis A, Anastasiou-Nana M, Roussos C, Nanas S: Physical exercise improves the peripheral microcirculation of patients with chronic heart failure. Journal of Cardiopulmonary Rehabilitation and Prevention 2009, 29:385-391.

47. Petersen A, Pedersen B: The anti-inflammatory effect of exercise. J Appl Physiology 2005, 98:1154-1162.

48. Fisher-Wellman K, Bloomer R: Acute exercise and oxidative stress: a 30 year history. Dynamic Medicine 2009, 8(1)

49. Fredriksson K, Rooyacckers O: Mitochondrial function in sepsis: Respiratory muscle versus leg muscle. Crit Care Med 2007, 35(9):S449-453.

50. Perme C, Chandrashekar R: Early mobility and walking program for patients in intensive care units: Creating a standard of care. AJCC 2009, 18(3):212-21

51. Bailey P, Thomsen GE, Spuhler VJ, Blair R, Jewkes J, Bezdjian L, Veale K, Rodriquez L, Hopkins RO: Early activity is feasible and safe in respiratory failure patients. Crit Care Med 2007, 35(1):139-145.

52. Hopkins R, Spulher V: Strategies for promoting early activity in critically ill mechanically ventilated patients. AACN Clin Issues 2009, 20(3):277-289.

53. Thomsen GE, Snow GL, Rodriguez L, Hopkins RO: Patients with respiratory failure increase ambulation after transfer to an intensive care unit where early activity is a priority. Crit Care Med 2008, 36(4):1119-1124.

54. Schweickert WD, Pohlman MC, Pohlman AS, Nigos C, Pawlik AJ, Esbrook CL, Spears L, Miller M, Franczyk M, Deprizio D, Schmidt GA, Bowman A, Barr R, Mccallister KE, Hall JB, Kress JP: Early physical and occupational therapy in mechanically ventilated critically ill patients: a randomised controlled trial. The Lancet 2009, 373:1874-1882.

55. Kress J, Polman A, O'Conner M, Hall J: Daily interruption of sedative infusions in critically ill patients undergoing mechanical ventilation. $N$ Engl J Med 2000, 342:1471-1477.

56. Griffiths RD, Palmer TE, Helliwell T, MacLennan P, MacMillan RR: Effect of passive stretching on the wasting of muscle in the critically ill. Nutrition 1995, 11:428-432.

57. Dellinger RP LM, Carlet JM, Bion J, Parker MM, Jaeschke R, Reinhart K, Angus DC, Brun-Buisson C, Beale R, Calandra T, Dhainaut JF, Gerlach H, Harvey M, Marini JJ, Marshall J, Ranieri M, Ramsay G, Sevransky J, Thompson BT, Townsend S, Vender JS, Zimmerman JL, Vincent JL, International Surviving Sepsis Campaign Guidelines Committee, American Association of Critical-Care Nurses, American College of Chest Physicians, American College of Emergency Physicians, Canadian Critical Care Society,
European Society of Clinical Microbiology and Infectious Diseases, European Society of Intensive Care Medicine, European Respiratory Society, International Sepsis Forum, Japanese Association for Acute Medicine, Japanese Society of Intensive Care Medicine, Society of Critical Care Medicine, Society of Hospital Medicine, Surgical Infection Society, World Federation of Societies of Intensive and Critical Care Medicine: Surviving Sepsis Campaign: International guidelines for management of severe sepsis and septic shock. Crit Care Med 2008, 36(1):1394-1396.

58. Knaus W, Draper $E$, Wagner D, Zimmerman J: APACHE II: a severity of disease classification system. Crit Care Med 1985, 13:818-829.

59. Vincent JL, M R, Takala J, Willatts S, De Mendonca A, Bruining H, Reinhart CK, Suter PM, Thijs LG: The SOFA (Sepsis related Organ Failure Assessment) score to describe organ dysfunction/failure. Intensive Care Med 1996, 22:707-710.

60. Charlson M, Pompe P, Ales K, Mackenzie C: A new method of classifying prognostic comorbidity in longitudinal studies: development and validation. J Chronic Dis 1987, 40(5):373-383.

61. Zeppos L, Patman S, Bernet S, Adsett J, Bridson J, Paratz J: Adverse physiological events in intensive care-is it an issue? Aust Journal of Physiotherapy 2007, 53:279-283.

62. Van Dillen L, Roach K: Reliability and validity of the acute care index of function for patients with neurologic impairment. Phys Ther 1988, 68:1098-1101.

63. Skinner E, Berny S, Warrillow S, Denehy L: Development of a physical function outcome measure (PFIT) and a pilot exercise training protocol for use in intensive care. Crit Care Resusc 2009, 11:110-115.

64. Roach K, Ally D, Finnerty B, Watkins D, Litwin B, Janz-Hoover B, Watson T, Curtis $K$ : The relationship between duration of physical therapy services in the acute care setting and change in functional status in patients with lower extremity orthopaedic problems. Phys Ther 1998, 78:19-24.

65. Rogers J, Ridley S, Chrispin P, Scotton H, Lloyd D: Reliability of the next of kins' estimates of critically ill patients' quality of life. Anaesthesia 1997, 52:1137-1143.

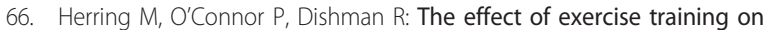
anxiety symptoms among patients. Arch Intern Med 2010, 170(4):321-331.

67. Kushner R: Bioelectrical impedance analysis: a review of principles and applications. Journal of the American College of Nutrition 1992, 11(2):199-209.

68. Lukaski HC, Bolonchuk WW, Hall CB, Siders WA: Validation of tetrapolar bioelectrical impedance method to assess human body composition. $J$ Appl Physiol 1986, 60(4):1327-1332.

69. Lingwood BE, Dunster KR, Ward LC: Cardiorespiratory monitoring equipment interferes with whole body impedance measurements. Physiol Meas 2005, 26(2):S235-S240.

70. Cornish B, Ward L, Thomas B, Jebb S, Elia M: Evaluation of multiple frequency bioelectrical impedance and Cole-Cole analysis for the assessment of body water volumes in healthy humans. Eur J Clin Nutr 1996, 50:159-164.

71. Levraut J, Ciebiera J, Chave S, Rabary O, Jambou P, Carles M, Grimaud D: Mild hyperlactatemia in stable septic patients is due to impaired lactate clearance rather than overproduction. Am J Respir Crit Care Med 1998, 157:1021-1026

72. Colier W, Meeuwsen I, Degens H, Oeseburg B: Determination of oxygen consumption in muscle during exercise using near infrared spectroscopy. Acta Anaesthesiol Scand 1995, 39(107 Suppl):151-155.

73. Strahovnik I, Podbregar M: Measurement of skeletal muscle tissue oxygenation in the critically ill. Signa Vitae 2008, 3(1):43-50.

74. Lindeboom J, Mathura K, Ramsoekh D, Harkisoen S, Aartman I, van den Akker $\mathrm{H}$, Ince $\mathrm{C}$ : The assessment of the gingival capillary density with orthogonal spectral polarization (OPS) imaging. ArchOral Biol 2006, 51:697-702.

75. De Backer D, Creteur J, Preiser J: Microvascular blood flow is altered in patients with sepsis. Am J Respir Crit Care Med 2002, 166:98-104.

76. Abate N, Trzeciak S: Is impaired capillary perfusion a marker of tissue hypoxia and a hallmark of incipient circulatory shock? Crit Care Med 2006, 34:566-567.

77. De Jonghe B, Sharshar T, Lefaucheur J, Authier F, Zaleski I, Boussarsar M, Charles C, Renaud E, Mesrati F, Carlet J, Raphae JC, Outin H, Bastuji-Garin S: Paresis acquired in the intensive care unit: A prospective multicenter study. JAMA 2002, 288(22):2859-2867. 
78. Lim S, Kim S, Park K, Kim S, Cho B, Yim M, Lee H: Effect of exercise on the mitochondrial DNA content of peripheral blood in healthy women. Eur $J$ Appl Physiol 2000, 82(5-6):407-412.

79. Burtin C, Langer D, Troosters T, Hermans G, Decramer M: Early exercise in critically ill patients enhances short-term functional recovery*. Crit Care Med 2009, 37(9):2499-2505

\section{Pre-publication history}

The pre-publication history for this paper can be accessed here: http://www.biomedcentral.com/1471-2253/11/21/prepub

doi:10.1186/1471-2253-11-21

Cite this article as: Kayambu et al: Early rehabilitation in sepsis: a prospective randomised controlled trial investigating functional and physiological outcomes The i-PERFORM Trial (Protocol Article). BMC Anesthesiology 2011 11:21.

\section{Submit your next manuscript to BioMed Central} and take full advantage of:

- Convenient online submission

- Thorough peer review

- No space constraints or color figure charges

- Immediate publication on acceptance

- Inclusion in PubMed, CAS, Scopus and Google Scholar

- Research which is freely available for redistribution

Submit your manuscript at www.biomedcentral.com/submit 